\title{
Dental health educators in general practice have small impact
}

\author{
Are dental health educators in general dental practice clinically and \\ cost-effective in improving oral health?
}

\begin{abstract}
Blinkhorn AS, Gratrix D, Holloway PJ, Wainwright-Stringer YM, Ward SJ, Worthington HV. A cluster randomised, controlled trial of the value of dental health educators in general dental practice. Br Dent J 2003; 195:395-400
\end{abstract}

Design A cluster randomised, controlled clinical trial of two years' duration.

Intervention Test group were given visits to a dental health educator over a 2-year period to counsel mothers of at-risk, pre-school children.

Outcome measure Caries prevalence of the children and dental health knowledge, attitudes and toothbrushing skills of the parents. The full costs of the exercise were kept throughout.

Results After 2 years, 271 (81\%) children and 248 (92\%) mothers remained in the study. There was an $18 \%$ difference in mean $\mathrm{dmft}$ between the groups in favour of the test group children but this was not statistically significant.

Conclusions The model tested of seconding a qualified dental health educator to general dental practices to counsel mothers of regularly attending, at-risk, young children failed to reveal a substantial improvement in dental health over a 2-year period. However, there were clear benefits in relation to dental health knowledge, attitudes and toothbrushing skills among these mothers.

\section{Commentary}

This is a very interesting and overall well-designed study to test whether targeted dental health education to pre-school children in a general practice setting can deliver worthwhile improvements in oral health. The children in the trial were assessed as likely to be at risk from caries before randomising into test and control groups. These are just the pre-school children that Healthcare Commissioning bodies as well as practitioners will want to reach with effective prevention methods.

However, the study suffers from two methodological drawbacks which could introduce the possibility of observer bias. These relate to the way in which the questionnaire on dental health knowledge and attitudes is administered and the method of collection of baseline caries levels. The authors discuss both since they could impact upon the findings which are weakly positive for provision of this type of dental health education in general practice.

The results are clearly disappointing and there is a good discussion as to why this may be the case. The authors' note that the two year duration of the study may not be sufficient to see a benefit with respect to caries progression. A further follow up of these children in time might see greater changes. However a longer duration would probably result in increasing drop-out from the study which then makes it difficult to be confident of the long term results. Watt et al ${ }^{1}$ argue that we need to look again how to evaluate the effectiveness of oral health promotion.

From a community health viewpoint it is discouraging that these regular attenders whom one might expect to benefit most from this type of intervention did not show appreciable health gains. Those seeking to improve the oral health of a local population will probably look to other methods to achieve this in the light of the results of this study. The systematic review of the effectiveness of oral health education by Kay and Locker ${ }^{2}$ mentioned in the paper only found a temporary effect on plaque accumulation but a sustained positive effect on knowledge. They found no effect of education programmes aimed at caries reduction which did not use fluoride.

\author{
Alan Lawrence \\ Chair, Evidence-based Dentistry Centre, Institute of Health \\ Sciences, Oxford
}

1. Watt RG, Fuller S, Harnett R, Treasure ET, Stillman-Lowe C. Oral health promotion evaluation - time for development. Commun Dent Oral Epidemiol 2001; 29:161-166.

2. Kay E J, Locker D. Is dental health education effective? A systematic review of current evidence. Commun Dent Oral Epidemiol 1996; 24:231-235.

Evidence-Based Dentistry (2004) 5, 15.

doi:10.1038/sj.ebd.6400239 